\title{
THERMODYNAMIC PROPERTIES OF PARTIALLY IONIZED HYDROGEN PLASMA
}

\author{
Maratbek Gabdullin', Tlekkabul Ramazanov², \\ Tomiris Ismagambetova ${ }^{3}$, Ainur Karimova ${ }^{4}$
}

\begin{abstract}
This paper considers dense partially ionized hydrogen plasma. The model of interaction between particles was used to study properties of plasma. Interaction potentials were obtained through the dielectric response function method. Effective potentials, taking into account the screening effects at large distances and the quantum-mechanical diffraction effect at small distances, were used to model the interaction between particles. Another effective screening potential was chosen to describe the charge interaction with neutral atoms. This potential takes into account the interaction between free charge and atomic nucleus with centrally symmetric distribution of the electron density. The degree of ionization was calculated through solving the system of Saha equations. Pair correlation functions were studied in the exponential approximation. Thermodynamic properties for hydrogen plasma were calculated using the effective potentials and obtained on their base pair correlation functions. Internal energy and equation of state of partially ionized hydrogen plasma were compared with the results from previous research. The results indicated that the difference observed with high values of parameters was due to increase in the concentration of atoms.
\end{abstract}

UDC Classification: 533.9 DOI: http://dx.doi.org/10.12955/cbup.v4.860

Keywords: plasma, potential, structural, thermodynamic.

\section{Introduction}

The object of this study is a non-ideal dense partially ionized hydrogen plasma.

Non-ideal plasma is determined by the major influence of interparticle interaction effects. The plasma becomes non-ideal when the average energy of interaction between the particles is comparable with a typical kinetic energy of thermal motion. The properties of such plasma are quite unusual and cannot be described by simple relations of the ideal gases and plasma theory.

The cores of giant planets, stars, and other astrophysical objects consist of non-ideal plasma (French et al., 2012). Non-ideal plasma is also a working body of pulsed thermonuclear reactors with inertial confinement of hot plasma. It involves powerful explosive magnetic and magneto-hydrodynamic generators; power plants and rocket engines with gas-phase nuclear reactors. It also comprises plasmachemical and microwave reactors; as well as plasma torches, and powerful sources of optical and $\mathrm{x}$-ray radiation (Fortov, \& Iakubov, 1999).

A model of interaction between particles in the plasma is necessary to correctly calculate the various plasma properties. There are two methods to develop effective interparticle interaction potential. The first consists of solving the generalized Poisson-Boltzmann equation, which is obtained from Bogolyubov's chain of equations for the distribution function in the phase space (Ecker, 1972). The second method involves using a method of dielectric constant function (Pines, \&Nozieres, 1966). The collective screening effect is needed to describe properties of non-ideal plasma. In addition, quantummechanical effects (such as diffraction) are required to study properties of dense plasma.

The model of interparticle interactions takes into account the collective effects at large distances and the quantum-mechanical effects at small distances, and has been used to study properties of non-ideal dense hydrogen plasma (Ramazanov, Moldabekov, \&Gabdullin, 2015).

\section{Method}

In the work of Ramazanov and Dzhumagulova (2002), the pseudopotential model for electron-electron and electron-ion interactions was obtained using the method of linear dielectric response. The

\footnotetext{
${ }^{1}$ Maratbek Gabdullin, NNLOT, al-Farabi Kazakh National University, Almaty, Kazakhstan, gabdullin @physics.kz

${ }^{2}$ Tlekkabul Ramazanov, Doctor of Physical and Mathematical Sciences, ITEP, al-Farabi Kazakh National University, Almaty, Kazakhstan, ramazan@physics.kz

${ }^{3}$ Tomiris Ismagambetova, ITEP, al-Farabi Kazakh National University, Almaty, Kazakhstan, miriael@mail.ru

${ }^{4}$ Ainur Karimova, al-Farabi Kazakh National University, Almaty, Kazakhstan, ainur.812@mail.ru
} 
analytical expression for the potential of the electron-electron and the electron-ion interaction has the following form:

$$
\Phi_{\alpha \beta}(r)=\frac{Z_{\alpha} Z_{\beta} e^{2}}{\sqrt{1-4 \lambda_{\alpha \beta}^{2} / r_{D}^{2}}}\left(\frac{e^{-B r}}{r}-\frac{e^{-A r}}{r}\right)
$$

Where:

$$
\begin{aligned}
& A^{2}=\frac{1}{2 \lambda^{2}}\left(1+\sqrt{1-\lambda_{\alpha \beta}^{2} / r_{D}^{2}}\right) \\
& B^{2}=\frac{1}{2 \lambda^{2}}\left(1-\sqrt{1-\lambda_{\alpha \beta}^{2} / r_{D}^{2}}\right) \\
& Z_{\alpha} e, Z_{\beta} e \text { are the atomic numbers of } \alpha, \beta \text { particles } \\
& \lambda_{\alpha \beta}=\hbar / \sqrt{2 \pi m_{\alpha \beta} k_{B} T} \text { is the thermal wave length } \\
& m_{\alpha \beta}=m_{\alpha} m_{\beta} /\left(m_{\alpha}+m_{\beta}\right) \text { is the reduced mass } \\
& r_{D}=\left(k_{B} T /\left(4 \pi e^{2} \sum_{j} n_{j} Z_{j}^{2}\right)\right)^{1 / 2} \text { is the Debye radius }
\end{aligned}
$$

Effective potential for ion-ion interaction was taken from the work of Ramazanov, Dzhumagulova, \&Gabdullin (2010):

$$
\Phi_{i i}(r)=\frac{Z_{i} Z_{i} e^{2}}{\sqrt{1-4 \lambda_{e i}^{2} / r_{D}^{2}}}\left(C_{1} \frac{e^{-A r}}{r}-C_{2} \frac{e^{-B r}}{r}\right)
$$

Where:

$$
\begin{aligned}
& C_{1}=\frac{1}{2}\left(1-\sqrt{1-\lambda_{e i}^{2} / r_{D}^{2}}\right) \\
& C_{2}=\frac{1}{2}\left(1+\sqrt{1-\lambda_{e i}^{2} / r_{D}^{2}}\right)
\end{aligned}
$$

These pseudopotentials consider the effects of short-range quantum-mechanical diffraction and longrange screening. The potential, taking into account the quantum diffraction effect, was used as a micro potential to construct the interaction model.

The screened potential obtained by Goldberger and Watson (1967) uses the dielectric response method. The used micro potential describes interaction between free charge and atomic nucleus and centrally symmetric distribution of the electron density. This approach was used as a potential of charge-atom interaction and involved the following equation:

$$
\Phi(r)=Z e^{2}\left(\frac{1}{r}+\frac{1}{a_{B}}\right) e^{-2 r / a_{B}},
$$

Where: $a_{B}$ is the Bohr radius. 
We used the exponential approximation to calculate pair correlation functions, as follows:

$$
g_{\alpha \beta}(r)=\exp \left(-\Phi_{\alpha \beta}(r) / k_{B} T\right)
$$

Where: $\Phi_{\alpha \beta}$ is the effective interaction potential of $\alpha$ and $\beta$ types of particles (Gabdullin et al., 2015).

Thermodynamic properties, such as internal energy and equation of state, were calculated using effective potentials (Equations 1, 7, \& 10) and were obtained using approximations of their base pair correlation functions (Equation 11), as follows:

$$
\begin{aligned}
& E=3 / 2 N k_{B} T-\pi \sum_{\alpha=i, e} n_{\alpha} \sum_{\beta=i, e} n_{\beta} \int_{0}^{\infty} g^{\alpha \beta}(r) \Phi^{\alpha \beta}(r) r^{2} d r \\
& P=n k_{B} T-\frac{2}{3} \pi \sum_{\alpha=i, e} n_{\alpha} \sum_{\beta=i, e} n_{\beta} \int_{0}^{\infty} \frac{\partial \Phi_{\alpha \beta}(r)}{\partial r} g_{\alpha \beta}(r) r^{3} d r
\end{aligned}
$$

Where: $n$ is the number of particles in the system (Ramazanov, Moldabekov, Gabdullin, \&Is magambetova, 2014).

The partial ionization was taken into account using the system of Saha equations (Gabdullin et al., 2015):

$$
\frac{n_{0}}{n_{e} n_{i}}=\frac{g_{0}}{g_{e} g_{i}} \exp \left(\frac{I-\Delta I}{k_{B} T}\right)
$$

Where: $g_{k}$ is the statistical weight, $I$ is the ionization potential, and $\Delta I$ is the lowering of the ionization potential.

\section{Results}

Figures 1 to 4 show the results of this study in comparison with the results of previous research. Figures 1 to 2 show the results for the internal energy and Figures 3 to 4 present the calculations for the equations of state. In all figures, the black squares represent the results of this study.

Figure 1 shows the results of Kraeft, Kremp, Ebeling, \&Röpke (1986; red circles), and Filinov et al. (2001; blue triangles) compared with those for this study (black squares). Figure 2 shows the results of Ebeling, Norman, Valuev, \&Valuev (1999; crossed triangles), Tanaka and Ichimaru (1985; triangles), and the Debye theory (dash-dotted line) compared with those of this study (black squares). Figure 3 shows the results of Knaup, Reinhard, \&Toepffer (2001; red circles), Militzer, \&Ceperley (2000; green triangles), and Militzer, \&Ceperley (2001; blue pentagrams) compared with this study's results (black squares). Figure 4 shows the results of Ebeling, Kraeft, \&Kremp (1976) for $A_{0}=0$ (red circles) and $\mathrm{A}_{0}=\pi / 2$ (green triangles) compared with the results of this study (black squares).

\section{Discussion}

The results of this study were compared with computer simulations of various approaches, such as the Montroll-Ward approximation (Kraeft, Kremp, Ebeling, \&Röpke, 1986), Monte-Carlo simulations (Militzer, \&Ceperley, 2001), the Green's function (Knaup, Reinhard, \&Toepffer 2001), and the data of Militzer, \&Ceperley(2001) that used the wave packet of molecular dynamics. A satisfactory agreement between the results of this study and those of other approximations was observed up to the point where the number density of particles was $10^{20 \mathrm{~cm}-3}$ (Figures 1 and 3). The agreement between different curves (Figures 2 and 4) was observed up to small values of the coupling parameter. These differences can be explained by the appearance of the atomic concentration of components in the system, which weakens the interaction in the system and leads to a decrease in absolute value of thermodynamic properties. 


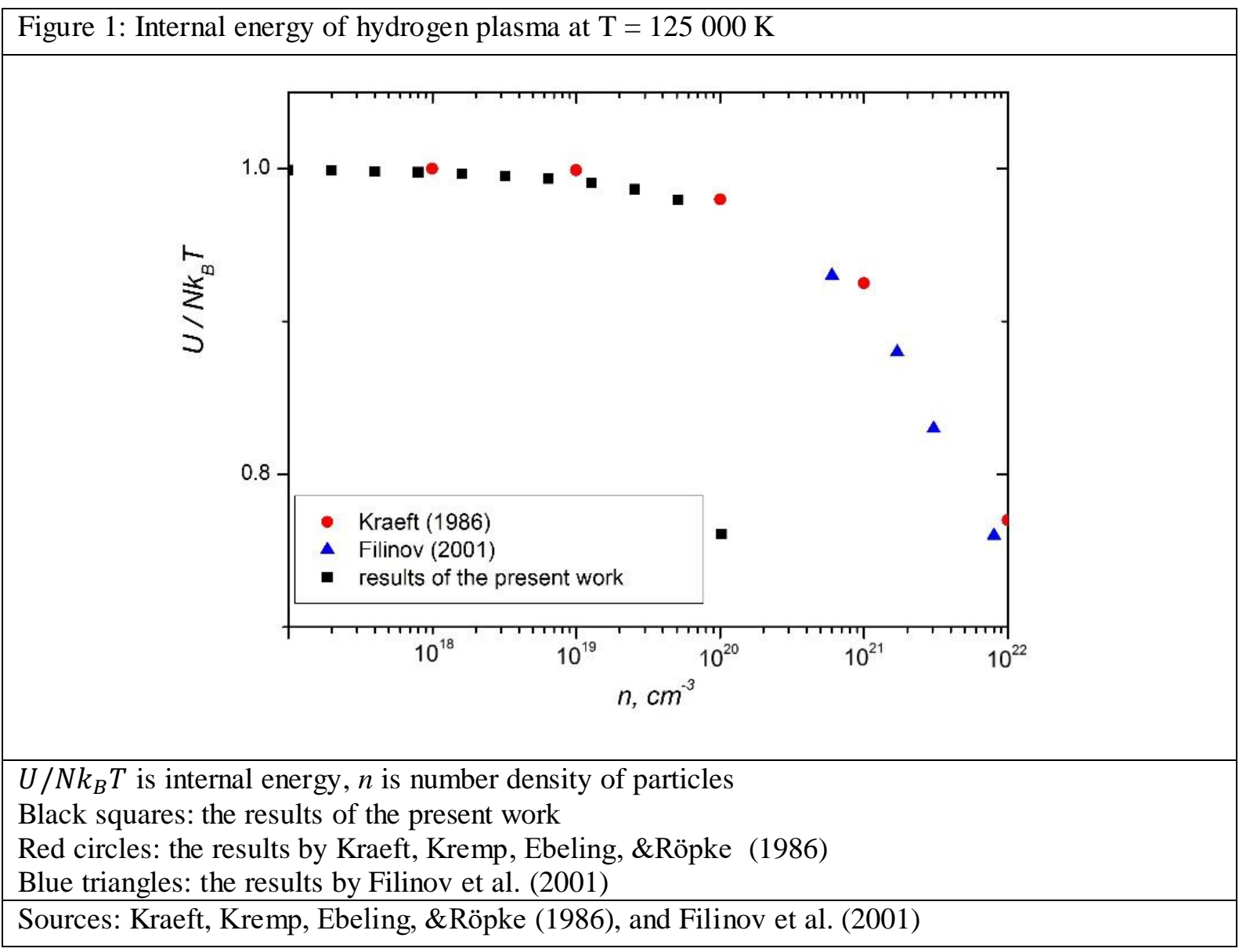

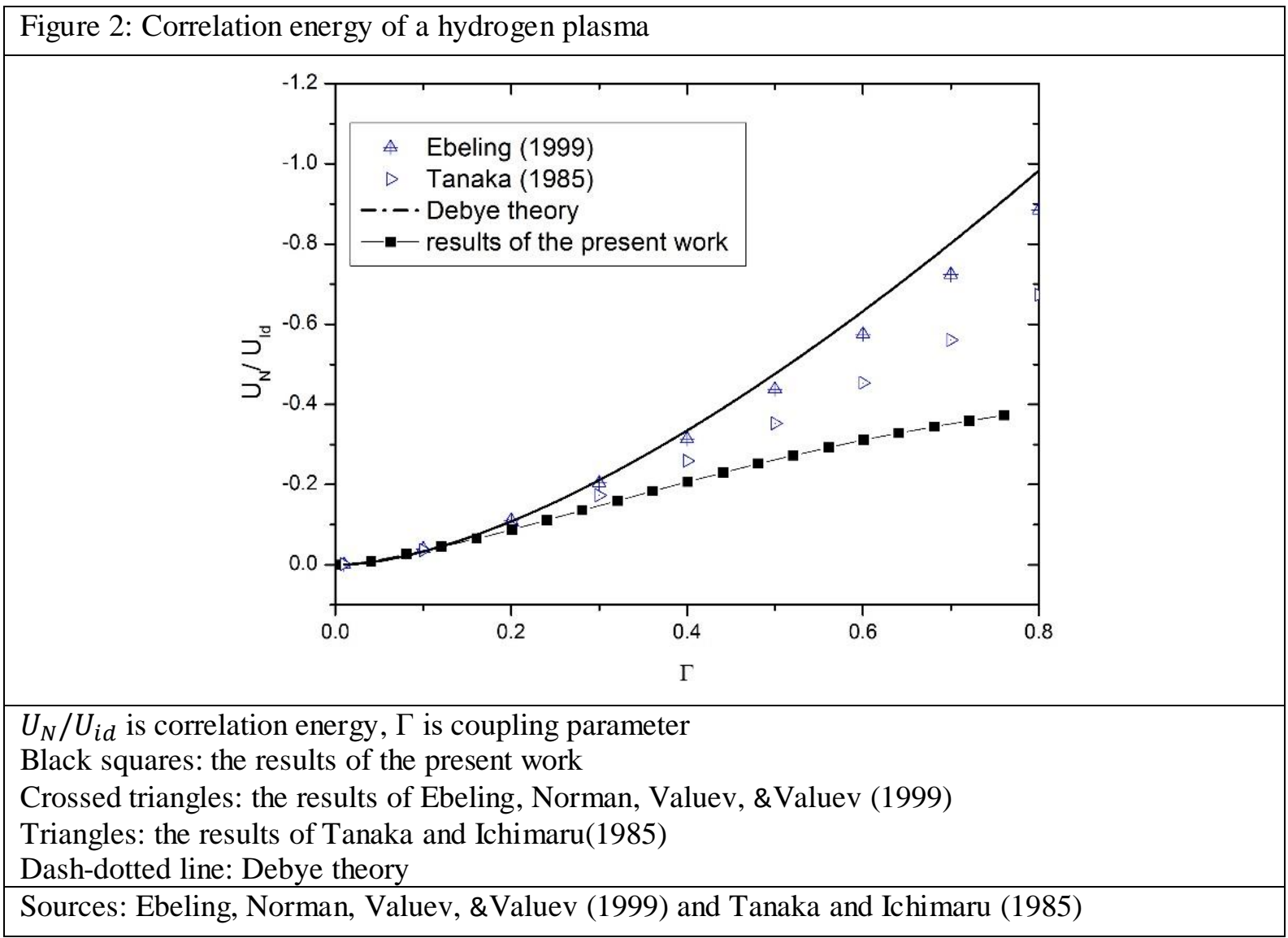




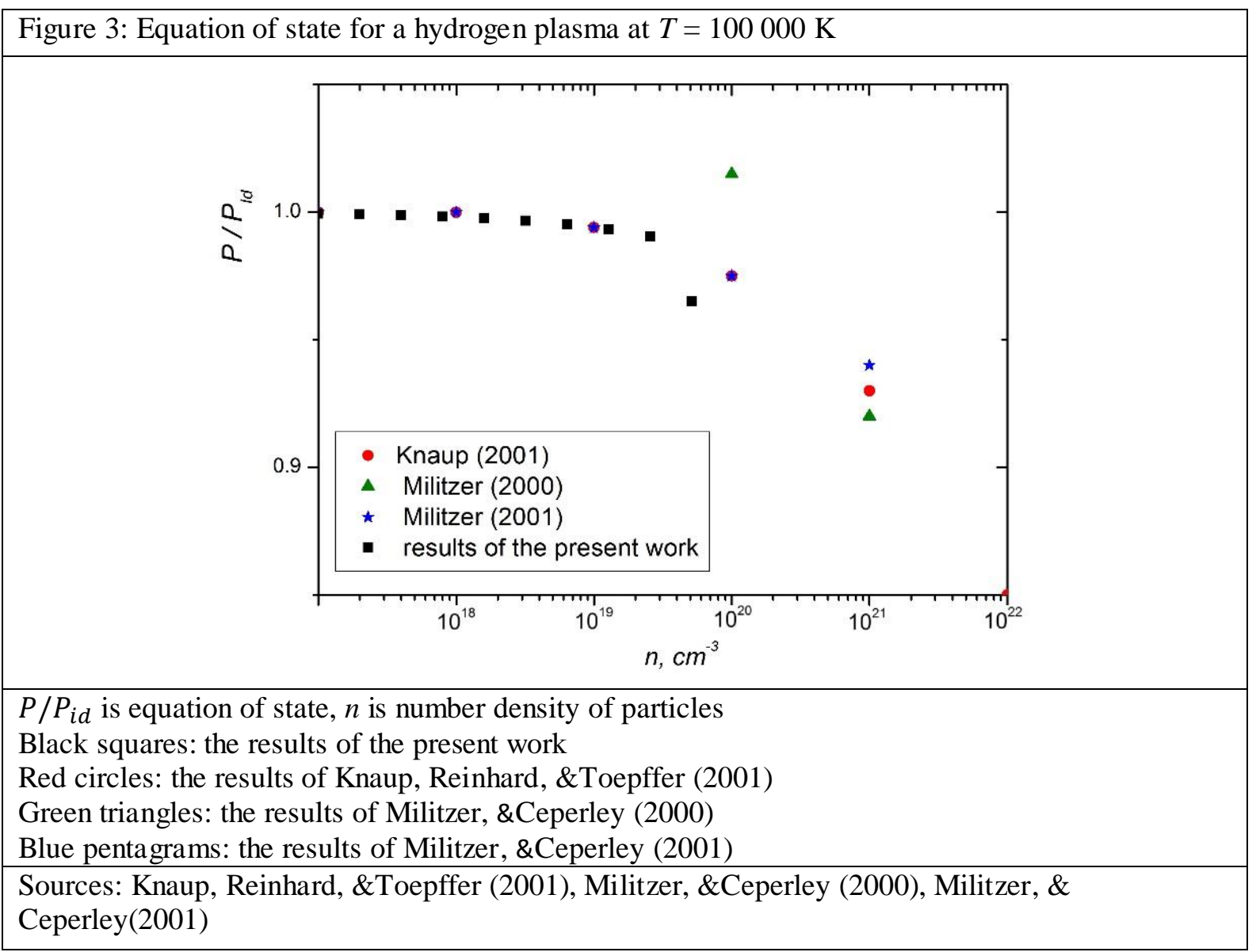

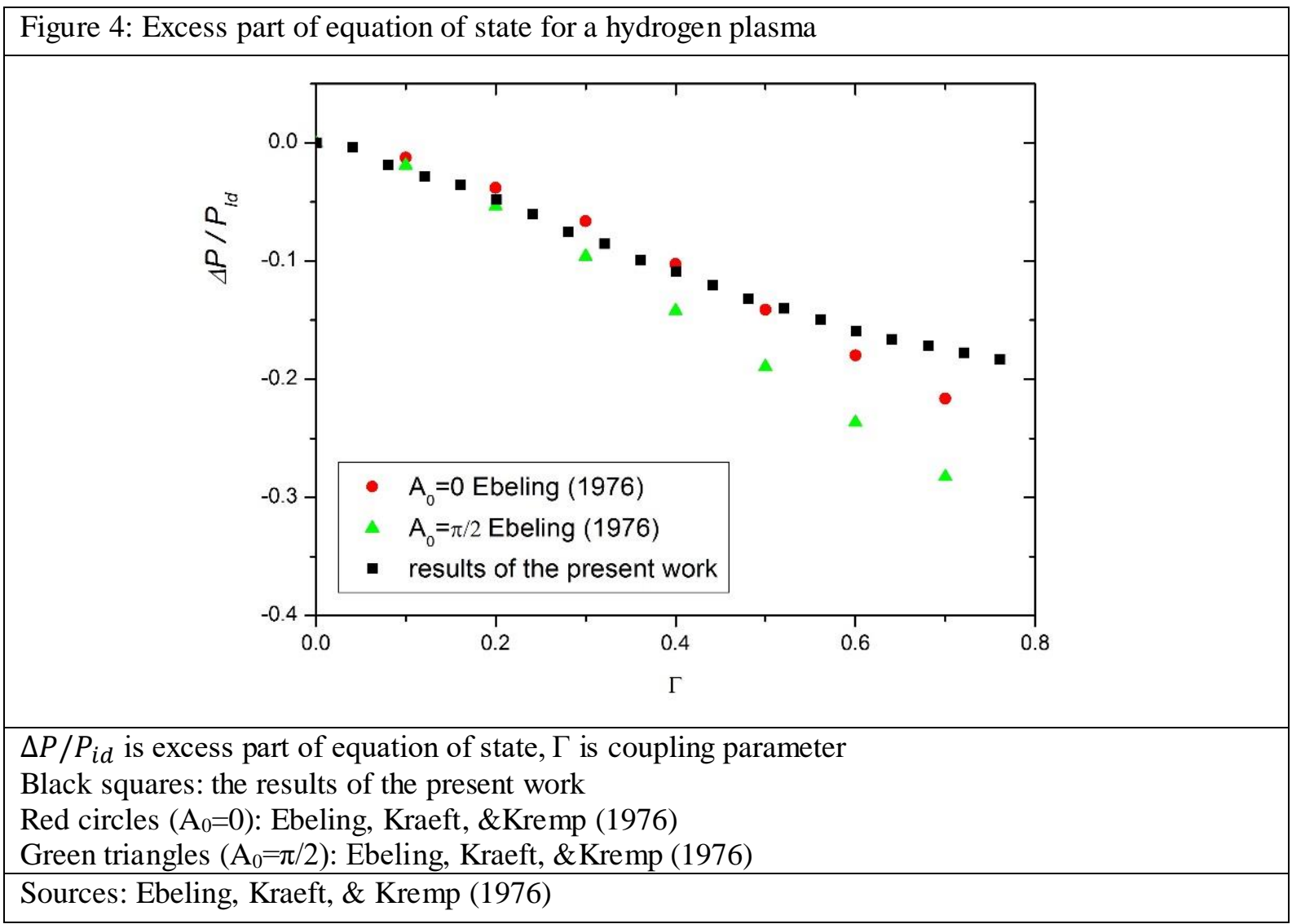




\section{Conclusion}

The internal energy and the equation for the state of partially ionized hydrogen plasma were calculated using the effective interaction potential, taking into account collective and quantum-mechanical effects and pair correlation functions. The results were compared with the data from previous research. Thermodynamic properties obtained in this work are in sound agreement with the results reported by other authors. The difference between the curves is associated with an increase in the concentration of atoms and a decrease in the concentration of free charge in the system.

\section{Acknowledgments}

This work has been supported by the Ministry of Education and Science of Kazakhstan under grant 3086/GF4.2016.

\section{References}

Ebeling, W., Kraeft, W. D., \& Kremp, D. (1976). Theory of bound states and ionization equilibrium. Berlin, AkademieVerlag.

Ebeling, W., Norman, G. E., Valuev, A. A., \& Valuev, I. A. (1999). Quasiclassical theory and molecular dynamics of twocomponent nonideal plasmas. Contrib. Plasma Phys., 39, 61.

Ecker, G. (1972). Theory of fully ionized plasmas. New York.

Filinov, V. S., Bonitz, M., Kremp, D., Kraeft, W. D., Ebeling, W., Levashov, P. R., \& Fortov, V. E. (2001). Path integral simulations of the thermodynamic properties of quantum dense plasma. Contrib. Plasma Phys., 41, 135.

French, M., Becker, A., Lorenzen, W., Nettelmann, N., Bethkenhagen, M., Wicht, J., \& Redmer, R. (2012). Ab initio simulations for the material properties along Jupiter's adiabat. Astrophys.J. Suppl. S., 202, 5.

Fortov, V. E., \& Iakubov, I. T. (1999). Physics of Nonideal Plasmas. World. Sci. Publ., London.

Gabdullin, M. T., Ramazanov, T. S., Muratov, M. M., Ismagambetova, T. N., Akhtanova, G. B., \& Goree, J. A. (2015).

Structural characteristics and equation of state of the complex plasmas. Contrib. Plasma Phys., 55(5), 366-372.

Goldberger, M., \& Watson, K. (1967). Theoryof collisions. Moscow.

Knaup, M., Reinhard, P. G., \& Toepffer, C. (2001). Wave packet molecular dynamics simulations of deuterium in the region of laser shock-wave experiments. Contrib. Plasma Phys., 41, 159.

Kraeft, W. D., Kremp, D., Ebeling, W., \& Röpke, G. (1986). Quantum statistics of charged particle systems. Berlin, Akademie-Verlag.

Militzer, B., \& Ceperley, D. M. (2000). Path integral Monte-Carlo calculations of the deuterium hugoniout. Phys. Rev. Lett., $85,1890$.

Militzer, B., \& Ceperley, D. M. (2001). Path integral Monte-Carlo simulations of the low-density hydrogen plasmas. Phys. Rev. E, 63, 066404.

Pines, D., \& Nozieres, Ph. (1966). The Theory of Quantum Liquids. New York.

Ramazanov, T. S., \& Dzhumagulova, K. N. (2002). Effective screened potentials of strongly coupled semiclassical plasma. Phys. Plasmas, 9 (9), 3758-3761.

Ramazanov, T. S., Dzhumagulova, K N., \& Gabdullin, M. T. (2010). Effective potentials for ion-ion and charge-atom interactions of dense semiclassical plasma. Phys. Plasmas., 17 (4), 042703.

Ramazanov, T. S., Moldabekov, Zh. A., Gabdullin, M. T., \& Ismagambetova, T. N. (2014). Interaction potentials and thermodynamic properties of two component semiclassical plasma. Phys. Plasmas, 21, 012706.

Ramazanov, T. S., Moldabekov, Zh. A., \& Gabdullin, M. T. (2015). Effective potentials of interactions and thermodynamic properties of a nonideal two-temperature dense plasma. Phys. Rev. E, 92, 023104.

Tanaka, S., \& Ichimaru, S. (1985). Parametrized equation of state for dense hydrogenetic plasma. Phys. Rev. A, $32,3756$. 